\title{
REPRODUCING
}

\section{THE FRESNEL-ARAGO EXPERIMENT TO ILLUSTRATE PHYSICAL OPTICS}

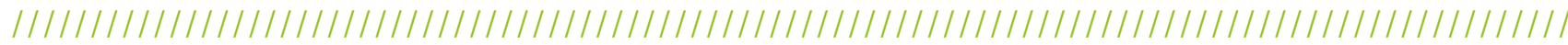

\section{Jérôme WENGER*, Benoît ROGEZ, Thomas CHAIGNE, Brian STOUT}

Aix Marseille Univ, CNRS, Centrale Marseille, Institut Fresnel, Marseille, France

* jerome.wenger@fresnel.fr

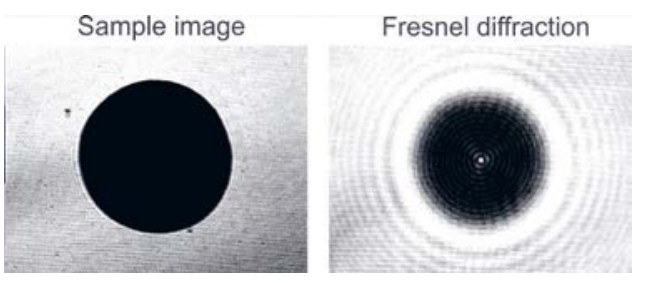

Is there a bright spot in the shadow of an opaque disk? Nearly 200 years ago, Augustin Fresnel and François Arago's remarkable answer to this question validated the wave theory of light and inaugurated the modern theories of diffraction. Today, their renowned experiment can be easily reproduced using lasers and cameras.

Far beyond its historical interest, the experiment is a versatile platform to illustrate the main concepts of optical physics, including diffraction, interference, speckle, and Fourier optics.

https://doi.org/10.1051/photon/202010421

This is an Open Access article distributed under the terms of the Creative Commons Attribution License (http://creativecommons.org/licenses/by/4.0), which permits unrestricted use, distribution, and reproduction in any medium, provided the original work is properly cited.

\section{INTRODUCTION: A BRIEF}

\section{HISTORICAL BACKGROUND}

ABOUT FRESNEL-ARAGO

\section{EXPERIMENT}

At the beginning of the $19^{\text {th }}$ century, Newton's corpuscular theory of light was hard pressed to explain Thomas Young's observations of interference fringes by light passing through double slits. Consequently, in 1818, the French Academy of Sciences launched a contest to reward an improved understanding of the properties of light. Augustin Fresnel authored one of two reports submitted to the Academy of Sciences [1]. Fresnel's essay notably developed a means to describe light diffraction using a wave theory of light based on Huygen's principal. The prize committee was principally composed of a renowned generation of scientists who favoured Newton's particle theory. Amongst their ranks was Siméon Poisson who thought he had debunked Fresnel's theory when he remarked that it predicts a bright

spot at the centre of the shadow of an opaque disk, which he held to be clearly false [3]. Fresnel realized however that shadows in everyday life generally lack the spatial

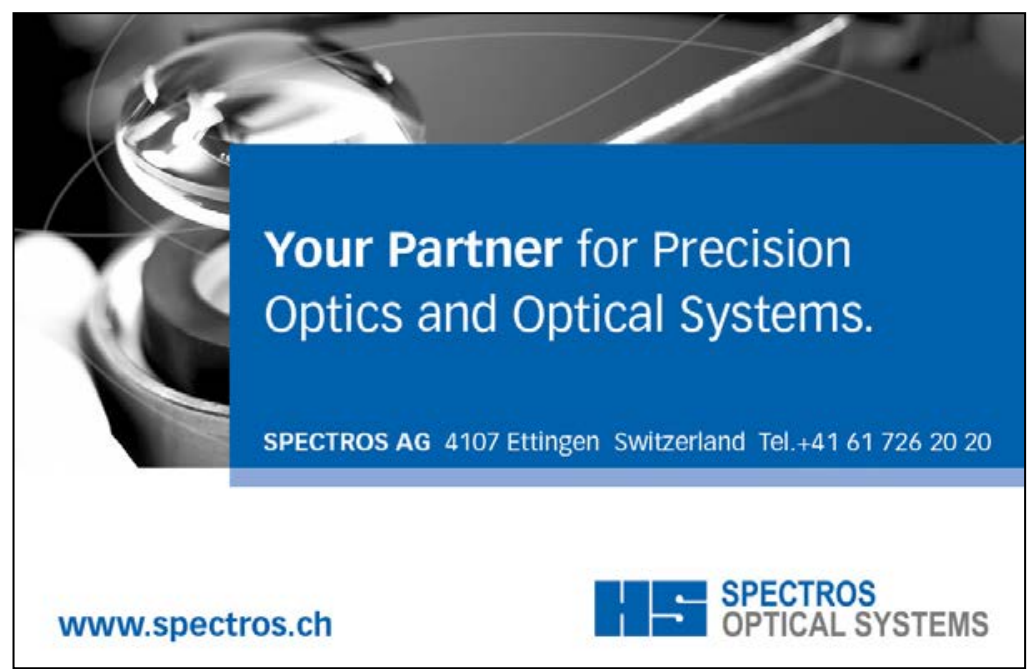




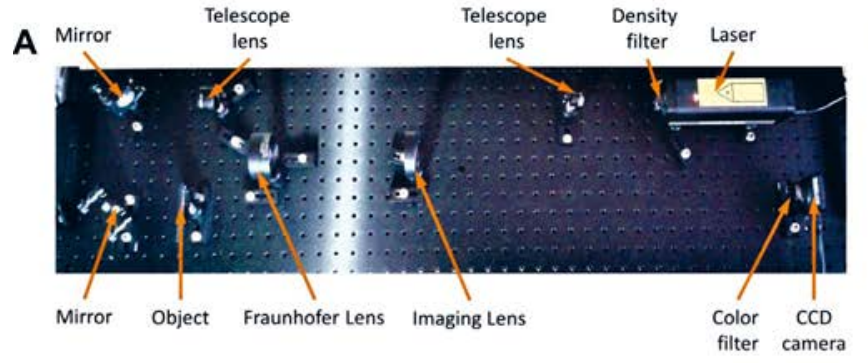

C

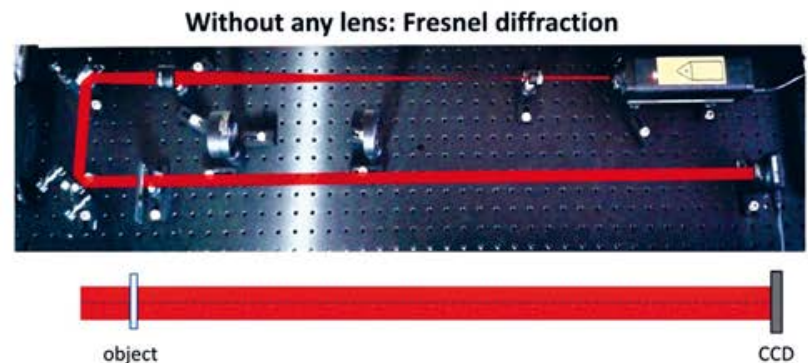

B

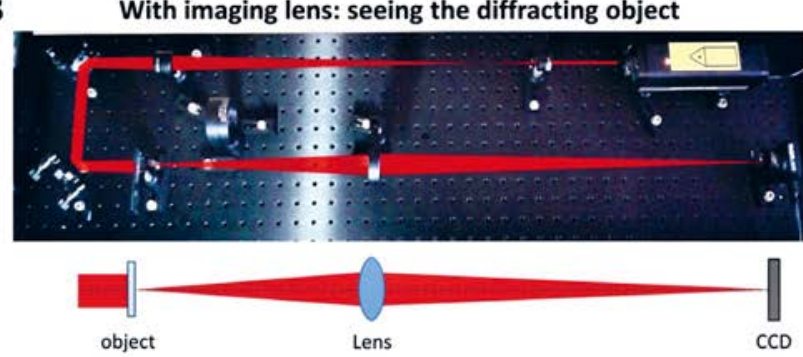

D

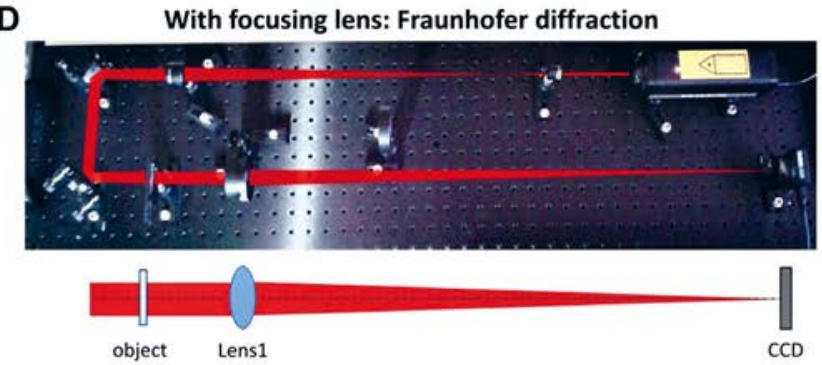

coherence and geometry required for such an observation. Fresnel, together with the committee president, François Arago, put Poisson's prediction to the test, and Arago announced to the world that there is indeed a bright spot in the shadow of a disk! It is difficult for scientific revolutions to be hailed overnight, and when the French Academy awarded Fresnel its prize in 1819, the official report highlighted the accuracy of the agreement between theory and experiment, while the word "wave" was mentioned only once. Fresnel's wave theory was almost immediately included in curriculums however, and its successes were of such magnitude that within only a few short years, the notion of light as particle emissions was relegated to the realm of historical anecdote until its spectacular revival by Einstein nearly a century later. Fresnel's prize marked only the beginning of the physical optics adventure, and several decades of discovery, mathematics and scientific revolution had to pass before Fresnel's intuitions and hypotheses were all firmly vindicated. In modern times, Fresnel's theory of light diffraction is now understood as an approximation to more complete theories, but its practicality is such that its use is still widespread.
Figure 1.

Experimental demonstration setup (A), with its different operation modes for imaging the sample (B), direct Fresnel diffraction (C) and Fraunhofer diffraction (D).

Now, more than 200 years later, using lasers and cameras, we can easily reproduce this historical experiment which played such a crucial role in our understanding of the properties of light.

\section{EXPERIMENTAL SETUP}

This modernized version of the Fresnel-Arago experiment is built on a $120 \times 30 \mathrm{~cm}^{2}$ breadboard for ease in handling (Fig. 1A). The light source is a $0.8 \mathrm{~mW}$ compact HeNe laser at $632.8 \mathrm{~nm}$, whose output is further attenuated, by a neutral density filter, down to $0.2 \mathrm{~mW}$. This power provides adequate visualization of the light beam while being low enough to ensure correct eye safety without requiring any specific laser safety equipment for the teacher or the students.

The laser beam is laterally expanded $16 \times$ using a telescope made of two lenses in order to reach a beam diameter of around $10 \mathrm{~mm}$ enabling correct visualization. The sample is placed directly on the laser beam path, held by two clips (Thorlabs FH2) which provide a versatile approach to move the sample during the live demonstrations. The camera is a USB-powered monochrome CMOS device (Thorlabs DCC1545M) whose sensor is placed in the light beam with no further optics. In order to operate under ordinary light conditions, the camera is equipped with a $610 \mathrm{~nm}$ long pass color filter to block most of the visible light from the room while transmitting the red laser light.

Fresnel diffraction by the sample object at finite distance is viewed directly on the camera (Fig. 1C). A supplementary biconvex lens of $200 \mathrm{~mm}$ focal length on a flippable mount can be used to image the sample on the camera (Fig. 1B). Additionally, another lens can be added to observe large distance Fraunhofer diffraction patterns (Fig. 1D). In this latter case, the lens is positioned so that the camera lies in the image focal plane of the lens. We used a $750 \mathrm{~mm}$ focal length, again on $90^{\circ}$ flip lens mount to allow inserting and removing the lens so as not to require optical alignment during demonstrations. Further details about the optical setups, including complete references of the components used can be found in Ref. [2]. 


\section{The most versatile Wavefront Sensor}

François Arago, put Poisson's prediction to the test, and Arago announced to the world that there is indeed a bright spot in the shadow of a disk!

\section{EXPERIMENTS \\ TO BE PERFORMED}

The demonstration setup allows to visually demonstrate major concepts of physical optics: diffraction, interferences, speckle, Fourier optics... Table 1 summarizes the main experiments that can be performed. Online videos on the Youtube channel of Institut Fresnel [3] show some examples of experiments recorded live. A principal common feature is to frequently switch from directly visualizing the sample (using the imaging lens, Fig. 1B) to observing the diffraction shadow (removing the imaging lens to be in Fresnel diffraction conditions, see Fig. 1C). Below, we briefly describe the different experiments and the sample preparation, focusing on simple methods that anyone can reproduce without specific tools.

The Fresnel-Arago diffraction experiment aims at observing the central bright spot in the shadow of an opaque disk. To fabricate the opaque disks, we deposited small dots of water-soluble black paint on a clean microscope glass slide using a thin brush or the tip of a needle. It takes a bit of trial and error to find the right consistency of the water-diluted black paint (paint for model making works well), but once the right consistency is achieved, nearly circular droplets of diameters from 0.5 to $2 \mathrm{~mm}$ are readily obtained. For the experiments, different disk diameters can be compared, showing that larger disks produce smaller Fresnel-Arago spots. Putting the disk

Table 1.

Demonstration experiments and main concepts illustrated with this setup.

\begin{tabular}{|c|c|}
\hline PHYSICAL OPTICS CONCEPT & TEASING TEXT FOR \\
\hline $\begin{array}{l}\text { Diffraction by a disk: } \\
\text { Fresnel-Arago spot }\end{array}$ & $\begin{array}{l}\text { This demonstration reproduces the historical } \\
\text { Fresnel-Arago experiment and explores the shadow } \\
\text { of an opaque disk, answering the question: is there } \\
\text { light in the center of a shadow from a black disk? }\end{array}$ \\
\hline $\begin{array}{l}\text { Diffraction by a hole: } \\
\text { the Airy disk }\end{array}$ & $\begin{array}{l}\text { What is the shadow of a single hole milled in an } \\
\text { opaque screen? The most studied case of diffraction } \\
\text { is illustrated here experimentally using simple } \\
\text { optical elements. }\end{array}$ \\
\hline $\begin{array}{l}\text { Young's double } \\
\text { slits interferences }\end{array}$ & $\begin{array}{l}\text { Can adding light to light create shadow? This } \\
\text { demonstration shows that this can indeed be the } \\
\text { case, reproducing Young's historical double slit } \\
\text { interferences experiment, which is now the most } \\
\text { studied interference configuration. }\end{array}$ \\
\hline $\begin{array}{l}\text { Random interferences } \\
\text { and Speckle }\end{array}$ & $\begin{array}{l}\text { What is the shadow of multiple scatterers } \\
\text { illuminated by a coherent laser beam? This } \\
\text { demonstration illustrates the principle of speckle } \\
\text { formation by multiple random interferences. }\end{array}$ \\
\hline Strioscopy and Fourier optics & $\begin{array}{l}\text { Mastering optics allows you to filter an image even } \\
\text { before it reaches the camera! This demonstration } \\
\text { illustrates the principle of strioscopy, which is a } \\
\text { specific application of Fourier optics. }\end{array}$ \\
\hline
\end{tabular}




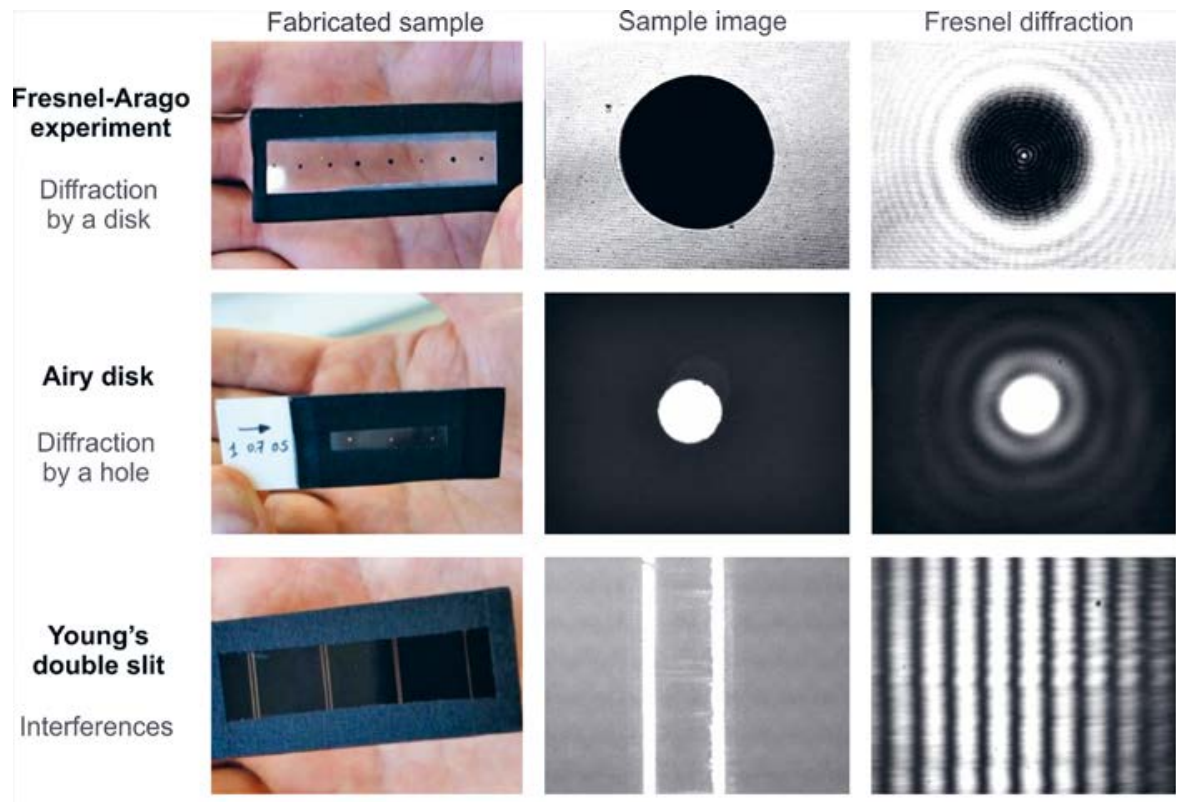

sample on a rail and moving it away from the camera gives nice results. This simple experiment allows one to travel through disk's shadow and observe the growth of the Fresnel diffraction pattern.

The same setup allows one to visualize the diffraction by a single hole; by far the most studied example of diffraction. To fabricate this sample, a thin plastic sheet was painted in black to make an opaque screen. Circular holes of diameters from 0.5 to $2 \mathrm{~mm}$ were then drilled manually using drill bits bought at a local hardware store. The effect of different hole diameters on the diffraction pattern can be illustrated. The Airy diffraction pattern is readily visible, and is important to discuss the resolution in optical microscopy. By moving the sample closer to the camera $(\sim 1-2 \mathrm{~cm})$, we can also observe dark regions at the centre of the bright spot, showcasing spectacular additional diffraction patterns.

Young's double slit interferences are also frequently used to discuss the interference phenomenon. To fabricate the single and double slits, a cleaned microscope glass slide was covered with black spray paint. Next, the paint layer was scratched using the tip of a needle guided by a ruler. Nearly parallel slits can easily be obtained this
Figure 2.

Examples of samples and experimental results for three selected demonstrations.

way, with an interslit distance below 1 $\mathrm{mm}$. This experiment illustrates how interferences lead to some regions having increased light intensity while in others it falls to nearly zero. Students can also see how changing the distance between slits affects the interfringe separation: narrower inter-slit distances yielding larger separation between fringes.

Supplementary experiments involve speckle formation and random interferences. Here, the sample consists of butterfly scales randomly deposited on a glass microscope slide, although virtually any multiple scattering media would produce similar conceptual results. In this experiment the sample is placed on the rail just in front of the camera (visualizing the shadow from each individual

\section{RÉFÉRENCES}

[2] B. Rogez, T. Chaigne, J. Wenger, arXiv 2002.03743 (2020) scatterer) then translating the sample to increase the sample-camera distance, the shadows of each scatterer progressively interfere until a complete random interference pattern (speckle) is obtained.

For the strioscopy experiment, the sample is a prepared microscope slide (slice of rat intestine in our case). The image is formed on the camera, then (keeping the imaging lens) a black disk (the same as for Fresnel-Arago experiment) is inserted in the lens back focal plane (200 $\mathrm{mm}$ away from the lens) to block the ballistic transmitted light and filter high spatial frequencies. As a consequence, the image contrast is inverted, and fine details stand out more prominently. This illustration of Fourier optics demonstrates how image formation is affected by the optical system, without involving computer processing.

\section{CONCLUSION}

The reproduction of a famous experiment of the early $19^{\text {th }}$ century turns out to be a rich and versatile platform to illustrate and demonstrate the main concepts of optical physics. Diffraction, interferences, speckle, image formation, Fourier optics and strioscopy can be shown in a didactic manner. Using modern optical elements, building this system and performing these experiments is nowadays quite easy [2]. Additionally, short videos illustrating the experiments described here can be found on the Youtube channel of Institut Fresnel. With a supplementary discussion of the coherence concept in optics, we hope that this modern homage will help to stimulate the next generation of young engineers, scientists and teachers.

[1] A.J. Fresnel, Guvres Complètes I (Paris: Imprimerie impériale, 1868)

[3] https://www.youtube.com/playlist?list=PLOqUzSRKRNCAuZZm4YLSkl1OHMKwCMTge 


\section{SEDI•ATI \\ fibres optiques}

\section{BRINGING LIGHT TO YOUR CUSTOMIZED, COMPLEX OR EXTREME ENVIRONMENT IS OUR CHALLENGE!}

Since 1972, SEDI-ATI Fibres Optiques designs and builds turn-key solutions based on optical fibers to enable you to bring photons in any environment, whatever your constraints are!

With 50 highly skilled and qualified professionals, a strong R\&D team, an ISO 9001 and ISO 13485 certified quality system, SEDIATI has in house resources required to adapt, integrate and qualify an intrinsically fragile material such as the optical fiber, to a wide variety of complex, harsh and even extreme environments. Two clean rooms are available as well as essential factory equipment such as two evaporation chambers, spectrometer, OTDR, special plasma glass bench, precision turning machine, pressure test bench, helium leak bench...

SEDI-ATI is very active on many demanding markets characterized by critical and extreme physical parameters such as temperatures close to $-273^{\circ} \mathrm{C}$ or above $600{ }^{\circ} \mathrm{C}$, pressures close to 5000 bars in detonation, or radiation levels up to $100 \mathrm{M}$ Gray. Besides, such parameters must often be combined and turn our challenges into real feats!

\section{YOU ARE AT THE HEART OF OUR SUCCESS}

Through our long-term relationship with our customers, we are proud to contribute to many pioneering projects in the fields of energy (LMJ, RJH, ITER), astronomy (MOONS, WEAVE), medical (NANOPTiX) and many other confidential projects. With the help of our customers, we thrive in many applications such as cryogenics for cyber security, quantum-optics, IR spectrometry, very high temperatures encountered in engine or furnace tests, as well as fiber-optic sensors deployed in the reactor core of a nuclear power plant.

\section{ANY EXISTING OPTICAL FIBER AT YOUR FINGERTIPS}

Our permanent stock of more than a hundred references of optical fibers allow us to offer various types of products:

- Bulkhead and inline fiber-optic hermetic feedthroughs.

- Achromatic multimode couplers and WDMs.

- Custom fiber optic assemblies, bundles, arrays and octopus.

- Fiber-optic spools for tethered vehicles.

- Special patch cords, cables, and connectors.

Fiber optic medical devices.

\section{SHARE YOUR NEEDS WITH US!}

SEDI-ATI is an agile company, creating sustainable and longterm value for its customers. We are always ready to listen to your requests and needs, to offer you optimal solutions and technical advice. So do not hesitate to consult us!

\section{CONTACT}

Pascal SLOBADZIAN / Sales Director

contact@sedi-ati.com / www.sedi-ati.com

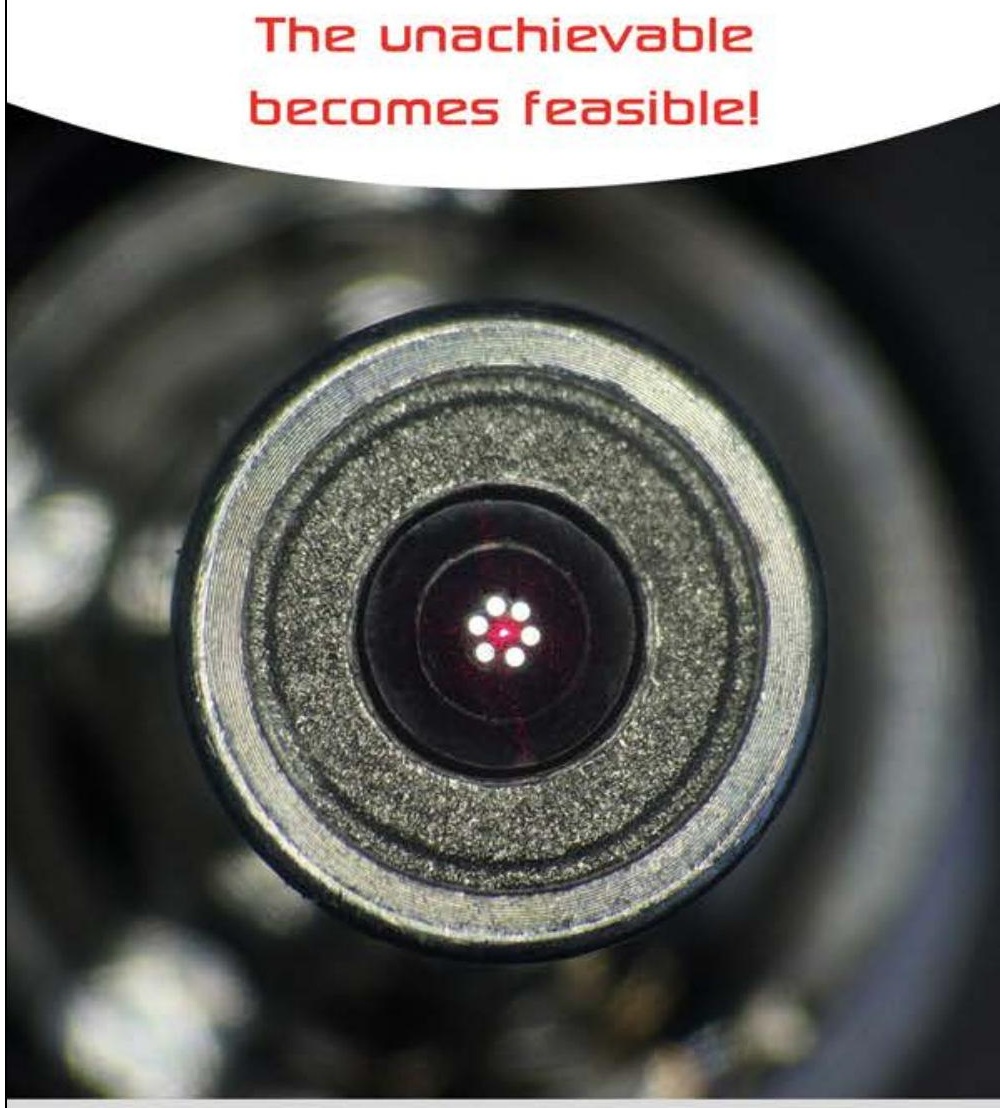

\section{A world first in the nuclear field!}

For the first time in history, a fiber-optic sensor has successfully demonstrated operation for a safety function in a nuclear environment.

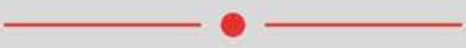

\section{Cryogenic cold}

Fiber-optic links and feedthroughs designed for quantum photonics applications, have demonstrated operation below $1.5 \mathrm{~K}$ and $10^{-11}$ high-vacuum.
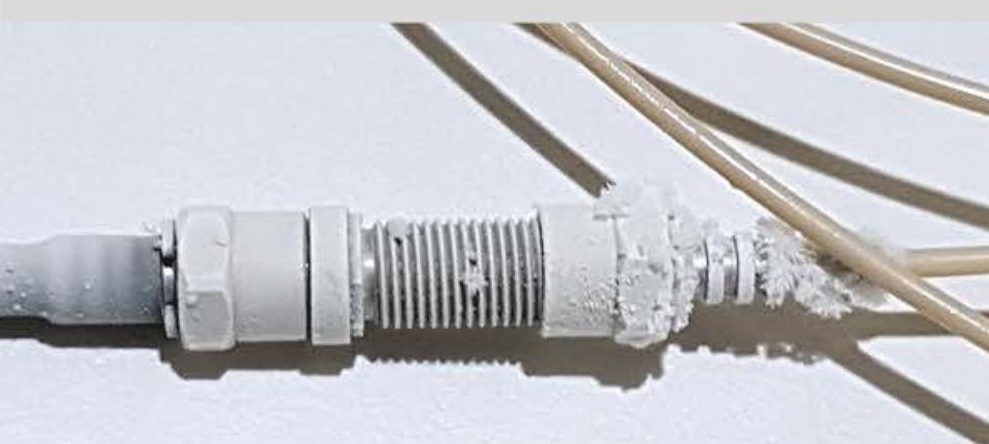

Share your dreams with us! 